\title{
EPIDEMIOLOGIA E FATORES DE RISCOS RELACIONADOS À INTOXICAÇÃO ALIMENTAR CAUSADA POR CLOSTRIDIUM BOTULINUM: UMA REVISÃO NARRATIVA
}

\author{
EPIDEMIOLOGY AND RISK FACTORS RELATED \\ TO FOOD INTOXICATION CAUSED BY \\ ClOSTRIDIUM BOTULINUM: A NARRATIVE REVIEW
}

\author{
Mirelle Costa Pignata-Viana', Juliana Silva Santos², \\ Pablo Teixeira Viana ${ }^{3}$
}

\begin{abstract}
RESUMO
O botulismo é uma doença resultante da ação de uma toxina produzida pelo Clostridium botulinum. Devido à sua gravidade e alta mortalidade é considerado um problema de saúde pública. Nesta revisão apresentamos os principais fatores de riscos associados à intoxicação alimentar provocada pelo Clostridium botulinum, bem como realizamos um levantamento epidemiológico sobre o botulismo alimentar e infantil. A busca bibliográfica considerou as bases de dados Scielo, Medline, Lilacs e PubMed. Foram selecionados artigos originais e relatos de caso publicados em inglês, espanhol e português, incluindo publicações dos últimos dez anos. A partir das análises dos títulos, resumos e artigos, um total de 26 artigos foram incluídos nesta revisão. Verificou-se predomínio de $54 \%$ dos casos referentes ao botulismo alimentar, dos quais aproximadamente $58 \%$ confirmaram a ocorrência da toxina tipo A; e 35\% referente ao botulismo infantil. Na literatura consultada os principais sintomas, relacionados ao botulismo alimentar, identificados foram: visão turva, vômito, paralisia flácida, náuseas, tontura, diplopia, dificuldade respiratória, disatria, disfagia, fraqueza muscular, boca seca, ptose e cefaleia. Dentre as principais fontes de contaminação, $65 \%$ das publicações selecionadas identificaram as conservas como principal causa do botulismo alimentar. Embora o mel (42\%) seja a única fonte registrada de alimento veiculador do agente causador do botulismo infantil, alguns relatos na literatura (25\%) associaram à doença com a inalação de poeira contendo esporos do Clostridium botulinum, bem como o uso de plantas medicinais (25\%). Os sintomas mais comuns observados na literatura foram: constipação dificuldade respiratória e dificuldade de sucção. Apesar de vários relatos na literatura acerca das duas doenças, o botulismo ainda é muito subnotificado dado ao diagnóstico muitas vezes equivocado, ressaltando-se a importância do diagnóstico precoce no tratamento da doença pelos profissionais de saúde, bem como a disponibilidade de informações relevantes para a investigação epidemiológica de doenças de notificação compulsória. Os dados apresentados também demonstram a importância de sensibilizar a população dos principais riscos e medidas de prevenção, já que a maioria dos casos relatados está relacionada a práticas inadequadas de preparo dos alimentos.
\end{abstract}

Palavras-chave: Botulismo; esporos; lactentes; neurotoxina; toxina alimentar

\section{ABSTRACT}

Botulism is a disease resulting from the action of a toxin produced by Clostridium botulinum. Because of its severity and high mortality, it is considered a public health problem. In this review, we present the main risk factors associated with food poisoning caused by Clostridium botulinum, as well as an epidemiological survey on foodborne and infant botulism. A bibliographic search was conducted in SciELO, MEDLINE, LILACS and PubMed databases. Original articles and case reports published in English, Spanish and Portuguese in the past ten years were selected. After analyzing titles, abstracts and articles, 26 articles were used in this review. In total, 54\% of the cases were related to foodborne botulism, of which approximately $58 \%$ had confirmed
Clin Biomed Res. 2019;39(2):161-170

1 Grupo de Pesquisa em Biotecnologia do Semiárido, Centro Universitário (UNIFG). Guanambi, BA, Brasil.

2 Faculdade de Farmácia, Centro Universitário (UNIFG). Guanambi, BA, Brasil.

3 Programa de Pós-graduação em Zootecnia, Universidade Federal da Bahia (UFBA). Salvador, BA, Brasil.

Autor correspondente: Mirelle Costa Pignata- Viana mirellepignata@hotmail.com Centro Universitário (UNIFG) Av. Pedro Felipe Duarte, 4911. 46430-000, Guanambi, BA, Brasil. 
type A botulism, and $35 \%$ were related to infant botulism. In the literature consulted, the main symptoms related to foodborne botulism were blurred vision, vomiting, flaccid paralysis, nausea, dizziness, diplopia, respiratory distress, dysarthria, dysphagia, muscle weakness, dry mouth, ptosis and headache. Among the sources of contamination, $65 \%$ of the published studies reported home-canned foods as the main cause of foodborne botulism. Although honey (42\%) is the only reported food source for the agent causing infant botulism, some reports in the literature $(25 \%)$ associated the disease with inhalation of dust containing Clostridium botulinum spores, as well as use of medicinal plants (25\%). The most common symptoms observed in the literature were constipation, difficulty breathing and difficulty suckling. Although several reports on the two forms of the disease exist, botulism remains under-reported because of often incorrect diagnosis. Thus, early diagnosis is important for an adequate treatment provided by health professionals, as well as availability of relevant information for the epidemiological investigation of notifiable diseases. The data presented in this study also demonstrate the importance of raising people's awareness to main risks and prevention measures, as most reported cases were related to inadequate food preparation practices.

Keywords: Botulism; spores; infants; neurotoxin; food toxin

O botulismo é uma doença resultante da ação de uma toxina produzida pelo Clostridium botulinum, normalmente decorrente da ingestão de alimentos contendo a toxina pré-formada. Caracteriza-se como uma doença grave, de evolução aguda, e que provoca alterações digestivas e neurológicas, podendo levar a falência respiratória seguida de óbito. O Clostridium botulinum é uma bactéria gram-positiva, esporulada e encontra-se naturalmente em diversos ambientes, como solo, água, mel, pólen, legumes frescos e especiarias, se desenvolvendo bem em condições de anaerobiose e elevada atividade de água ${ }^{1,2}$.

São descritos na literatura sete tipos de Clostridium botulinum indicados pelas letras A, B, C, D, E, F e G, que se diferenciam pelas características antigênicas da neurotoxina que produzem, embora possuam ação farmacológica similar. Sendo as do tipo $A, B, E$ e $F$ as mais tóxicas para o ser humano, a do tipo $G$ associada a causas de morte súbita, enquanto os tipos C e D estão relacionados ao botulismo em aves e mamíferos ${ }^{3}$.

Em condições de anaerobiose, elevada atividade de água $(0,94-0,98)$ e $\mathrm{pH}$ superior a 4,5 , a forma vegetativa do Clostridium botulinum produz uma toxina potencialmente fatal. A destruição dos esporos botulínicos ocorre, de modo geral, à temperatura de esterilização $\left(120^{\circ} \mathrm{C}\right.$ por 30 minutos). No entanto, alimentos ácidos $(\mathrm{pH}<4,5)$ podem ser processados em temperaturas abaixo de $100{ }^{\circ} \mathrm{C}$, pois o meio ácido possibilita a destruição do microrganismo, não havendo produção de toxina, embora os esporos possam estar presentes e sobreviver ao tratamento térmico ${ }^{4}$.

Esse microrganismo foi descrito inicialmente no século XVIII, na Europa, após a investigação de um surto que atingiu vários consumidores de salsicha ${ }^{5}$.
Atualmente, são descritas três formas de botulismo: alimentar ou clássico, por ferida e infantil ou lactente ${ }^{2,6,7}$. O botulismo alimentar ocorre pela ingestão da toxina pré-formada, enquanto os demais tipos são decorrentes da infecção, proliferação e produção de toxinas em feridas ou no trato gastrointestinal ${ }^{8}$.

Em todos os tipos de botulismo a causa da doença é comum, observando-se sintomas semelhantes como náuseas, vômitos, paralisia flácida e dificuldade respiratória. A toxina é absorvida no lúmen intestinal e ativamente é transportada para a circulação sistêmica. Ao atravessar a corrente sanguínea, a toxina é transportada para as terminações neuromuscular onde bloqueia o lançamento de acetilcolina, produzindo o seu efeito tóxico. Devido ao seu peso molecular (150 kDa), esta não atravessa a barreira hematoencefálica e os sintomas são limitados ao sistema nervoso periférico ${ }^{9}$.

O botulismo possui distribuição mundial e envolve casos isolados ou surtos familiares. Em função de sua alta letalidade é considerada um problema de saúde pública ${ }^{8}$. No Brasil, de 1999 a 2006 foram confirmados 32 casos de botulismo. Destes, $31 \mathrm{de}$ origem alimentar com taxa de letalidade de $28 \%$, segundo a Coordenação de Doenças de Transmissão Hídrica e Alimentar ${ }^{10}$. No interior da Bahia foram confirmados, em 2002, dois casos de botulismo alimentar envolvendo uma criança de sete anos e uma adolescente de 13 anos, após a ingestão de embutido (chouriça) fabricado no interior do Estado do Paraná, comercializado nos municípios envolvidos ${ }^{6}$.

A alta taxa de mortalidade está relacionada ao diagnóstico clínico tardio e a insuficiência respiratória é uma das principais causas de morte. O botulismo, ainda muito subnotificado, é confundido com outras doenças no diagnóstico do paciente, como Síndrome 
de Guillain-Barré, Síndrome de Miller-Fisher e Miastenia Gravis, retardando o tratamento do paciente. No Brasil, desde 2001, a notificação de casos suspeitos tem sido compulsória. Tal medida facilita o desenvolvimento de investigações epidemiológicas e sanitárias, além de implementar novas medidas sanitárias para o controle e prevenção de novos surtos ${ }^{11}$.

O diagnóstico laboratorial do botulismo têm sido importante para esclarecer os casos da doença, a fim de confirmar o diagnóstico clínico e identificar a toxina. No entanto, por ser demorado, o tratamento depende dos cuidados intensivos de suporte com base na manifestação clínica ${ }^{11}$, como bloqueio neuromuscular, provocando paralisia simétrica flácida, aguda e descendente com dificuldade visual, alterações na fala e na deglutição².

O diagnóstico clínico envolve a anamnese do paciente, fazendo-se necessário investigar sobre os alimentos consumidos, o tempo entre a ingestão e o surgimento dos sintomas, existência de outros casos e fontes comuns de ingestão e caracterização dos sinais e sintomas apresentados. A Avaliação neurológica consiste na verificação dos movimentos, avaliando o déficit de força, em diferentes músculos; comprometimento da musculatura facial, ocular e bulbar; prejuízo de reflexos profundos e verificação da consciência do paciente ${ }^{2,3}$.

Assim que é diagnosticado, o botulismo deve ser tratado em Unidade de Terapia Intensiva, com ventilação mecânica em casos mais graves e deve ser realizada a administração da antitoxina botulínica para eliminar a toxina circulante, podendo parar a progressão da paralisia e reduzir a duração da doença ${ }^{5}$. Segundo Jalda et al. ${ }^{12}$, com o tratamento adequado, a mortalidade da doença em adultos é inferior a 10\% e a maior recuperação da força muscular ocorre nos três primeiros meses de infecção, embora alguns pacientes podem levar até um ano para recuperar a força e função ventilatória normais.

A prevenção da doença se dá desde o controle na produção até os cuidados no consumo do alimento ${ }^{12,13}$. Ademais, as conservas caseiras oferecem um maior risco para a ocorrência das toxinas do C. botulinum, em virtude da manipulação inadequada na preparação dos alimentos e ausência de oxigênio, uma vez que são acondicionados em embalagens hermeticamente fechadas ${ }^{7}$. A ingestão de alimentos, principalmente produtos artesanais preparados sem o devido tratamento, contendo a toxina pré-formada pelo Clostridium botulinum ocasiona uma das principais doenças de origem alimentar ${ }^{10}$.

O botulismo infantil, por sua vez, está relacionado à absorção da toxina produzida in vivo no intestino da criança ${ }^{14}$. Trata-se de uma doença associada à Síndrome de Morte Súbita do Recém-Nascido, em função do consumo de mel contendo esporos botulínicos, o qual é germinado no intestino grosso e produz a toxina in vivo ${ }^{12}$. O tipo de toxina e a quantidade de esporos ingeridos pela criança determinam a gravidade do quadro clínico, devendo-se evitar o consumo de mel no primeiro ano de vida ${ }^{1}$.

Diante do exposto, objetivou-se com a realização dessa revisão de literatura, estudar os principais fatores de riscos associados à intoxicação alimentar provocada pelo Clostridium botulinum, bem como realizar um levantamento epidemiológico sobre o botulismo alimentar e botulismo infantil.

\section{MÉTODOS}

Foi realizada uma revisão narrativa de caráter descritivo sobre a epidemiologia e fatores riscos relacionados à intoxicação alimentar causada pelo Clostridium botulinum. As buscas na literatura foram conduzidas de maneira exploratória nas principais bases de dados.

A seleção dos artigos foi realizada por meio de pesquisa bibliográfica em quatro bases de dados distintas: Scielo, Medline, Lilacs e PubMed. A consulta foi composta de dois domínios básicos. O primeiro relacionado ao botulismo alimentar ou infantil e o segundo, ao microrganismo em questão (Clostridium botulinum). Nesse contexto, a seguinte estratégia genérica de busca foi obtida: (botulismo alimentar OU botulismo infantil) E (Clostridium botulinum). O procedimento de pesquisa foi concluído em fevereiro de 2019.

Adotou-se como método de pesquisa específica a construção para uso no PubMed, adaptando-se em seguida para as demais bases de dados, conforme segue: "food botulism"[All Fields] OR neurotoxin[All Fields] OR (("food"[MeSH Terms] OR "food"[All Fields])AND ("toxins, biological"[MeSH Terms] OR ("toxins"[All Fields] AND "biological"[All Fields]) OR "biological toxins"[All Fields] OR "toxin"[All Fields])) OR (childlike[All Fields] AND ("botulism"[MeSH Terms] OR "botulism"[All Fields])) OR ("spores"[MeSH Terms] OR "spores"[All Fields]) OR ("infant”[MeSH Terms] OR "infant"[All Fields]) AND "clostridium botulinum"[All Fields].

Os artigos foram selecionados em duas fases: na primeira fase, os estudos decorrentes da pesquisa bibliográfica foram avaliados, com base em seus títulos e resumos. Enquanto, a segunda etapa foi realizada a partir da avaliação dos textos completos, conforme os critérios de inclusão e exclusão, com posterior leitura interpretativa e redação do artigo.

Foi definido como critério de elegibilidade para inclusão dos artigos (originais e relatos de caso) os estudos que trataram da construção (pesquisa), identificação ou discussão dos termos: investigação 
epidemiológica, patogenia do microrganismo, alimentos de riscos, investigação microbiológica e toxicológica de amostras clínicas e de alimentos, produção de neurotoxina botulínica, medidas de controle da doença, características clínicas e tratamento da doença. Foram excluídos na revisão narrativa i) artigos em duplicata; ii) textos em línguas diferentes do português, inglês e espanhol; iii) artigos relacionados ao botulismo em animais ou botulismo de lesões (feridas); iv) estudos e pesquisas de anais de congressos, monografias, dissertações, teses e boletins informativos; e v) não estar entre os anos de publicação (2008 até 2019). Optou-se por esse período de publicação, a fim de se obter dados mais recentes sobre o tema abordado.

\section{RESULTADOS}

As estratégias de busca permitiram a identificação de 1019 artigos. Destes, oito encontravam-se replicados nas bases de dados pesquisadas. A partir das análises dos títulos, resumos e artigos, um total de 1011 trabalhos foram excluídos por vários motivos: abordagem de tópicos irrelevantes; não apresentação de resumos e/ou artigos na íntegra; pesquisas escritas em idiomas distintos dos selecionados; abordagem de botulismo em animais ou por feridas e fora do ano de publicação determinado, permanecendo para análise final da revisão narrativa 26 artigos (Figura 1).

Encontra-se na Tabela 1 as especificações de cada um dos artigos selecionados, quanto ao tipo de botulismo, fontes suspeitas de contaminação e principais sintomas da doença. $\mathrm{Na}$ análise dos 26 trabalhos incluídos na presente revisão, verificou-se predomínio de publicações provenientes dos Estados Unidos, Brasil e Argentina, ambos com aproximadamente $15 \%$. Do total de trabalhos pesquisados, 14 artigos são referentes ao tema botulismo alimentar (54\%), dos quais aproximadamente $58 \%$ confirmam a ocorrência da toxina tipo A; nove relatam o botulismo infantil ( $35 \%$ ) e três abordam as duas doenças (11\%).

$\mathrm{Na}$ literatura consultada os principais sintomas, relacionados ao botulismo alimentar, identificados

Tabela 1: Publicações incluídas na revisão de literatura, segundo o tipo de botulismo, fontes suspeitas de contaminação e principais sintomas da doença.

\begin{tabular}{|c|c|c|c|c|c|c|}
\hline Botulismo & Fontes suspeitas & $N^{0}$ de $\operatorname{casos}^{a}$ & Sintomas & Pais & Ano & Autores \\
\hline Alimentar & $\begin{array}{l}\text { Conservas, } \\
\text { embutidos, } \\
\text { pescados e queijo }\end{array}$ & 26 & Paralisia flácida. & Brasil & 2008 & Cereser et al. ${ }^{4}$ \\
\hline Infantil & Mel & - & - & Brasil & 2008 & Ragazani et al. ${ }^{14}$ \\
\hline Infantil & $\begin{array}{l}\text { Infusão de folhas } \\
\text { de orégano }\end{array}$ & 1 & $\begin{array}{l}\text { Desidratação, febre } \\
\text { e níveis pressóricos } \\
\text { elevados, depressão } \\
\text { respiratória, redução } \\
\text { dos reflexos em } \\
\text { forma simétrica e } \\
\text { descendente, ptose e } \\
\text { midríase. }\end{array}$ & Chile & 2008 & Córdova et al. ${ }^{1}$ \\
\hline Ambos & $\begin{array}{l}\text { Alimentos } \\
\text { processados em } \\
\text { casa, conservas e } \\
\text { mel }\end{array}$ & $309^{*}$ & $\begin{array}{l}\text { Náuseas, vômitos, dor } \\
\text { abdominal e diarreia. }\end{array}$ & Argentina & 2008 & Tornese et al. ${ }^{5}$ \\
\hline Alimentar & Suco de cenoura & 3 & $\begin{array}{l}\text { Neuropatias cranianas } \\
\text { e paralisia flácida. }\end{array}$ & $\begin{array}{l}\text { Estados } \\
\text { Unidos }\end{array}$ & 2008 & Sheth et al. ${ }^{15}$ \\
\hline Alimentar & $\begin{array}{l}\text { Pimenta em } \\
\text { conserva }\end{array}$ & 1 & $\begin{array}{l}\text { Cefaleia, visão turva, } \\
\text { diplopia, disartria, } \\
\text { náuseas e vômitos. }\end{array}$ & $\begin{array}{l}\text { Estados } \\
\text { Unidos }\end{array}$ & 2008 & Bilusic et al. ${ }^{16}$ \\
\hline Infantil & Poeira do ambiente & 2293 & $\begin{array}{l}\text { Constipação, } \\
\text { sonolência, paralisia } \\
\text { flácida, dificuldade de } \\
\text { sucção. }\end{array}$ & Itália & 2009 & $\begin{array}{l}\text { Fenicia e } \\
\text { Anniballi17 }\end{array}$ \\
\hline Infantil & Mel & 1 & $\begin{array}{l}\text { Constipação, fraqueza } \\
\text { muscular progressiva } \\
\text { e distúrbios } \\
\text { respiratórios. }\end{array}$ & Chile & 2009 & Arriagada et al. ${ }^{9}$ \\
\hline
\end{tabular}

aNúmero total de casos suspeitos de botulismo alimentar e botulismo infantil pesquisado na literatura; *34 casos suspeitos de botulismo alimentar e 70 casos suspeitos de botulismo infantil; ${ }^{* * 117}$ casos suspeitos de botulismo alimentar; ${ }^{* *} 1173$ casos suspeitos de botulismo alimentar e 275 casos suspeitos de botulismo infantil. 
Tabela 1: Continuação...

\begin{tabular}{|c|c|c|c|c|c|c|}
\hline Botulismo & Fontes suspeitas & $N^{0}$ de $\operatorname{casos}^{a}$ & Sintomas & Pais & Ano & Autores \\
\hline Infantil & $\begin{array}{l}\text { Flor de Tília } \\
\text { (utilizada como } \\
\text { sedativo natural na } \\
\text { forma de chá) }\end{array}$ & - & - & Argentina & 2009 & Bianco et al. ${ }^{18}$ \\
\hline Infantil & Mel e poeira & 456 & - & Argentina & 2009 & Sagua et al. ${ }^{19}$ \\
\hline Alimentar & $\begin{array}{l}\text { Bebida alcoólica } \\
\text { artesanal }\end{array}$ & 4 & $\begin{array}{l}\text { Visão turva, disartria, } \\
\text { disfagia, falta de ar e } \\
\text { fraqueza muscular. }\end{array}$ & $\begin{array}{l}\text { Estados } \\
\text { Unidos }\end{array}$ & 2009 & Vugia et al. ${ }^{20}$ \\
\hline Ambos & $\begin{array}{l}\text { Carne em conserva, } \\
\text { patê de fígado de } \\
\text { porco, creme de } \\
\text { tofu, torta de frango, } \\
\text { conserva caseira de } \\
\text { pescado e mel. }\end{array}$ & $117^{* *}$ & $\begin{array}{l}\text { Cefaleia, tontura, } \\
\text { paralisias de nervos } \\
\text { cranianos, paralisia } \\
\text { muscular descendente } \\
\text { flácida e simétrica. }\end{array}$ & Brasil & 2010 & Rowlands et al. ${ }^{11}$ \\
\hline Alimentar & Conservas & 66 & $\begin{array}{l}\text { Visão turva, tontura, } \\
\text { disfagia, disfonia, } \\
\text { diplopia, cefaleia, } \\
\text { vômito. }\end{array}$ & China & 2010 & Zhang et al. ${ }^{21}$ \\
\hline Alimentar & Azeitonas pretas & 3 & $\begin{array}{l}\text { Visão turva, diplopia, } \\
\text { disfagia, disatria, boca } \\
\text { seca, tontura, etc. }\end{array}$ & Escócia & 2010 & Swaan et al. ${ }^{22}$ \\
\hline Alimentar & Torta de frango & 3 & $\begin{array}{l}\text { Insuficiência } \\
\text { respiratória, tontura, } \\
\text { fraqueza muscular } \\
\text { progressiva, } \\
\text { sonolência e vômito, } \\
\text { etc. }\end{array}$ & Brasil & 2011 & Barboza et al. ${ }^{8}$ \\
\hline Alimentar & $\begin{array}{l}\text { Korma (molho } \\
\text { a base de curry, } \\
\text { creme, coco e } \\
\text { amêndoas). }\end{array}$ & 3 & $\begin{array}{l}\text { Visão turva, } \\
\text { dificuldade de } \\
\text { deglutição e paralisia } \\
\text { descendente. }\end{array}$ & Escócia & 2011 & Browning et al. ${ }^{23}$ \\
\hline Alimentar & $\begin{array}{l}\text { Pasta de azeitonas } \\
\text { verde }\end{array}$ & 2 & $\begin{array}{l}\text { Visão turva, tontura, } \\
\text { sonolência, fraqueza } \\
\text { muscular, etc. }\end{array}$ & França & 2011 & Pingeon et al. ${ }^{24}$ \\
\hline Infantil & Poeira do ambiente & 1 & $\begin{array}{l}\text { Paralisia flácida, } \\
\text { dificuldade de sucção } \\
\text { e respirar. }\end{array}$ & Finlândia & 2014 & Derman et al. ${ }^{25}$ \\
\hline Alimentar & $\begin{array}{l}\text { Conservas e } \\
\text { produtos de origem } \\
\text { animal }\end{array}$ & 321 & $\begin{array}{l}\text { Insuficiência } \\
\text { respiratória, cardíaca e } \\
\text { paralisia flácida. }\end{array}$ & & 2014 & $\begin{array}{l}\text { Juliano e } \\
\text { Cardoso }^{10}\end{array}$ \\
\hline Infantil & Poeira do ambiente & 1 & $\begin{array}{l}\text { Hipotonia, } \\
\text { irritabilidade, choro } \\
\text { fraco e constipação. }\end{array}$ & Costa Rica & 2014 & $\begin{array}{l}\text { Hernández-de } \\
\text { Mezerville et al. }{ }^{26}\end{array}$ \\
\hline Alimentar & $\begin{array}{l}\text { Adzuki-batto } \\
\text { (sopa de feijão } \\
\text { adzuki levemente } \\
\text { adocicada contendo } \\
\text { macarrão de trigo } \\
\text { embalado a vácuo). }\end{array}$ & 2 & $\begin{array}{l}\text { Ptose, boca seca, } \\
\text { visão turva, dificuldade } \\
\text { de respirar, náuseas, } \\
\text { vômito, paralisia } \\
\text { flácida. }\end{array}$ & Dinamarca & 2014 & Momose et al. ${ }^{27}$ \\
\hline Alimentar & Conservas & 3 & $\begin{array}{l}\text { Ptose, boca seca, } \\
\text { visão turva, dificuldade } \\
\text { de respirar, náuseas, } \\
\text { vômito, paralisia } \\
\text { flácida. }\end{array}$ & $\begin{array}{l}\text { Estados } \\
\text { Unidos }\end{array}$ & 2014 & Viray et al. ${ }^{28}$ \\
\hline
\end{tabular}


Tabela 1: Continuação...

\begin{tabular}{|c|c|c|c|c|c|c|}
\hline Botulismo & Fontes suspeitas & $\mathrm{N}^{\circ}$ de casos $^{\mathrm{a}}$ & Sintomas & Pais & Ano & Autores \\
\hline Alimentar & Costela defumada & 12 & $\begin{array}{l}\text { Diplopia, tontura, } \\
\text { disfagia, ptose, e } \\
\text { disartria, náuseas e } \\
\text { vômito. }\end{array}$ & Dinamarca & 2015 & Feng et al. ${ }^{29}$ \\
\hline Infantil & - & 1 & $\begin{array}{l}\text { Constipação e } \\
\text { abdome agudo. }\end{array}$ & Argentina & 2015 & Spinia et al. ${ }^{30}$ \\
\hline Alimentar & $\begin{array}{l}\text { Conserva de feijão } \\
\text { verde }\end{array}$ & 3 & $\begin{array}{l}\text { Diplopia, dor } \\
\text { abdominal difusa e } \\
\text { paralisia do nervo } \\
\text { craniano. }\end{array}$ & Espanha & 2016 & $\begin{array}{l}\text { Jalda } \\
\text { Sánchez et al. }{ }^{12}\end{array}$ \\
\hline Ambos & $\begin{array}{l}\text { Conservas, mel e } \\
\text { infusão de ervas }\end{array}$ & $1257^{* * *}$ & $\begin{array}{l}\text { Diplopia, dificuldade } \\
\text { de sucção, boca seca, } \\
\text { constipação, náuseas } \\
\text { e vômito. }\end{array}$ & Itália & 2017 & Anniballi et al. ${ }^{31}$ \\
\hline
\end{tabular}

\section{Busca nas bases de dados}

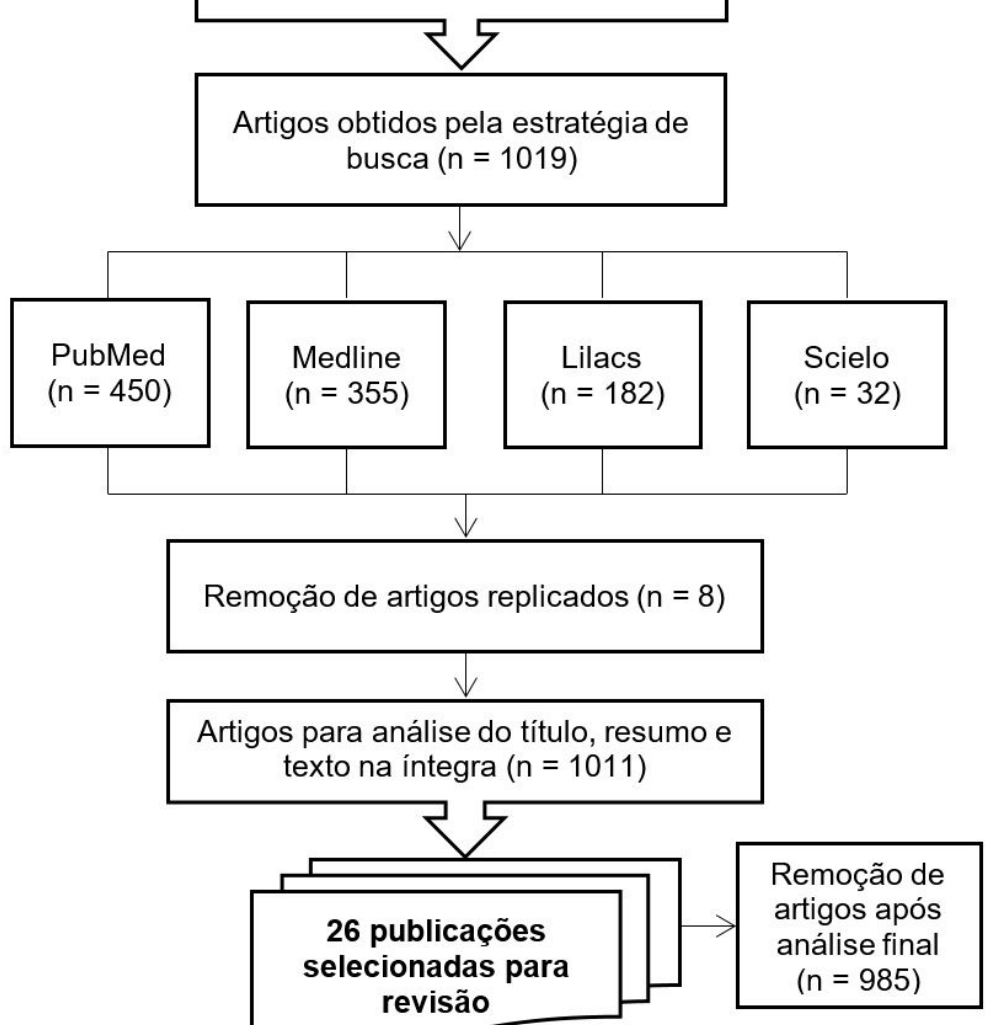

Figura 1: Diagrama de fluxo da revisão narrativa.

foram: visão turva $(n=8)^{16,20,21,22,23,24,27,28}$; vômito $(\mathrm{n}=8)^{5,8,16,21,27,28,29,31}$; paralisia flácida $(\mathrm{n}=7)^{4,10,11,15,23,27,28}$; náuseas $(\mathrm{n}=6)^{5,16,27-29,31}$; tontura $(n=5)^{11,21,22,24,29}$; diplopia $(n=5)^{12,16,21,22,29}$; dificuldade

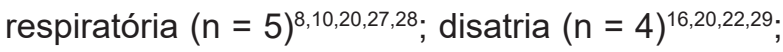
disfagia $(n=4)^{20,21,22,29}$; boca seca $(n=4)^{22,27,28,31}$; fraqueza muscular $(n=3)^{8,20,24}$; ptose $(n=3)^{27-29}$ e cefaleia $(n=3)^{11,16,21}$. Dentre as principais fontes 
de contaminação, 11 (65\%) das 17 publicações pesquisadas identificaram as conservas como principal causa do botulismo alimentar ${ }^{4,5,10-12,16,21-23,28,31}$.

Algumas das fontes potenciais mais reconhecidas de esporos de Clostridium botulinum causadores do botulismo infantil são: o mel $(42 \%)^{5,11,14,19,31}$; poeira $(33 \%)^{17,19,25,28}$ e ervas medicinais $(33 \%)^{1,18,19,31}$. Os sintomas mais comuns observados na literatura foram: constipação $(n=5)^{17,18,26,30,31}$; dificuldade respiratória $(\mathrm{n}=3)^{1,18,25}$ e dificuldade de sucção $(\mathrm{n}=3)^{17,25,31}$.

Quanto ao número total $(\mathrm{n}=4875)$ de casos suspeitos de botulismo descrito na literatura, observou-se maior frequência para o botulismo infantil ( $n=3099$ ), equivalente a $64 \%$ dos casos, contra $36 \%$ referente ao botulismo alimentar.

\section{DISCUSSÃO}

Neste estudo foi realizada uma revisão narrativa, com o objetivo de estudar a epidemiologia e os principais fatores de riscos associados à intoxicação alimentar provocada pelo Clostridium botulinum. Ao final da busca, 26 artigos atenderam aos critérios de inclusão e foram incluídos no estudo.

O botulismo é uma doença rara, potencialmente fatal, ocasionada pela ingestão da toxina pré-formada no alimento ${ }^{14}$. Os sintomas clínicos da doença aparecem após um período de incubação, o qual varia muito a depender do sorotipo e o grau de exposição à toxina ${ }^{29}$.

As fontes intoxicantes limitam-se ao tipo de botulismo. Os alimentos envolvidos nos surtos de botulismo variam conforme os hábitos de conservação e alimentação em diferentes países. Na Argentina, entre os anos de 1992 e 2003, foram notificados 34 casos de botulismo alimentar, onde a maioria está associada ao consumo de alimentos processados em casa ou conservas preparadas inadequadamente ${ }^{5}$.

Rowlands et al. ${ }^{11}$ realizaram uma investigação laboratorial de 117 casos suspeitos de botulismo notificados à Vigilância Sanitária, ocorridos no Brasil no período de janeiro de 2000 a outubro de 2008. Foram analisadas 193 amostras clínicas e 81 amostras de alimentos extraídas de vários estados brasileiros (Amazonas, Bahia, Ceará, Goiás, Maranhão, Mato Grosso, Mato Grosso do Sul, Minas Gerais, Pará, Paraná, Pernambuco, Rio de Janeiro, Rio Grande do Norte, Rio Grande do Sul e São Paulo). Observou-se a prevalência da toxina em 22 amostras clínicas e oito amostras de alimentos, tais como carne em conserva, patê de fígado suíno caseiro e industrializado, tofu, torta de frango e conserva caseira de pescado. Dentre os 117 casos suspeitos investigados, $27(23,1 \%)$ foram confirmados para botulismo, provocados pela toxina tipo $\mathrm{A}(66,7 \%)$, com alta taxa de letalidade $(34,2 \%)$.

Anniballi et al. ${ }^{31}$ observaram que do total de 1257 casos suspeitos de botulismo notificados, entre os anos de 1986 e 2015, 466 foram confirmados laboratorialmente, observando-se um aumento médio anual de 40 notificações e 15 confirmações. A maioria dos casos confirmados de botulismo alimentar ( $n=421)$ ocorreu em áreas rurais da Itália e, em especial, na região da Campânia. A maior prevalência dos casos nessas áreas justifica-se pela ampla disponibilidade de matéria-prima utilizada no preparo de conservas caseiras, aliado ao baixo custo de produção.

As conservas, frequentemente envolvidas em surtos de botulismo alimentar (Tabela 1), quando não esterilizadas adequadamente, oferecem condições favoráveis ao desenvolvimento do Clostridium botulinum, decorrente da atmosfera anaeróbica, além da presença de nutrientes, água em quantidade suficiente, ambiente com acidez baixa e livre de inibidores de crescimento $^{5}$, tais como o nitrito e nitrato (conservantes), usualmente empregado em produtos cárneos no controle do C. botulinum ${ }^{11}$.

Feng et al. ${ }^{29}$ relataram um surto familiar na China, decorrente do consumo de costela defumada. $O$ produto foi contaminado, provavelmente, durante o preparo, em função de manipulação inadequada, ausência de conservantes, além de tratamento térmico insuficiente (defumação a frio) para inativar os esporos botulínicos. De acordo com os autores, por meio das diretrizes da Food and Drug Administration (FDA), o método de defumação a frio não é recomendado devido aos riscos inerentes, uma vez que o rápido crescimento microbiano pode ocorrer na zona de temperatura utilizada. Além disso, os alimentos defumados a frio devem ser cozidos internamente a $160^{\circ} \mathrm{C}$ antes de serem consumidos, recomendando-se um binômio tempo-temperatura de 30 minutos a $121^{\circ} \mathrm{C}$ para destruição de esporos butolínicos.

Alimentos de baixa acidez são mais propícios ao desenvolvimento de Clostridium botulinum. Segundo Sheth et al. ${ }^{15}$, um suco de cenoura industrializado foi a provável causa do surto de botulismo na Geórgia, Flórida e Canadá. Embora o produto seja pasteurizado, a temperatura empregada ( $72-75^{\circ} \mathrm{C}$ por 15 a 20 segundos) não é suficiente para destruir os esporos botulínicos. Tais condições favorecem a germinação deste quando não apresenta barreira intrínseca, como o emprego de acidulantes e conservantes. O surto foi resultante da contaminação com C. botulinum tipo A, cuja temperatura mínima para crescimento é de $10^{\circ} \mathrm{C}$, com subsequente crescimento e produção de toxina. 
Na França, o botulismo tornou-se um problema de notificação compulsória desde 1986 e entre 2007 e 2009, 47 casos foram notificados às autoridades sanitárias, sendo 33 causados pela toxina tipo A e destes, três eram lactentes. Em setembro de 2011 foram notificados à Vigilância Sanitária da França dois surtos familiares de botulismo. Após investigação em amostras clínicas (soro, fezes e suco gástrico) e restos de alimentos consumidos pelas duas famílias, confirmou-se a presença da toxina botulínica tipo A em sete amostras de soro, dos nove pacientes acometidos, bem como na pasta de azeitonas verde artesanal, ingeridas em momentos distintos por ambas as famílias ${ }^{24}$.

Os esforços para reduzir a incidência da doença devem ser direcionados a medidas educacionais em saúde pública, a fim de proporcionar melhor compreensão acerca da gravidade do botulismo, baseando-se em precauções sanitários relevantes. Tal medida preserva a identidade cultural e a popularidade de alimentos tradicionalmente preparados em algumas regiões ${ }^{5}$.

Quanto ao botulismo infantil, esta doença é a forma mais comum de botulismo humano ${ }^{18}$, associada frequentemente ao consumo de mel contaminado por esporos do Clostridium botulinum, presentes no pólen coletado pelas abelhas ${ }^{25}$. Em estudo realizado por Ragazini et al. ${ }^{14}$, após avaliação de 100 amostras de méis comercializados nos Estados de São Paulo, Mato Grosso, Goiás, Ceará, Minas Gerais e Santa Catarina, observaram que $61 \%$ das amostras analisadas havia a presença de bactérias esporuladas. Dentre estas, $11 \%$ era do gênero Clostridium e destas, confirmaram-se $7 \%$ como sendo Clostridium botulinum. Segundo os autores, a presente investigação reforça a importância deste achado para a saúde pública, ressaltando-se que o mel é a única fonte registrada como veiculador do agente causador do botulismo infantil.

Segundo Arriagada et al. ${ }^{9}$, o botulismo é uma doença rara no Chile. No entanto, dentre os tipos o infantil é responsável pela maioria dos casos. No mundo, mais de 3000 casos de botulismo infantil foram identificados entre 1976 e 2007, com maior frequência nos EUA ${ }^{26}$. Entre 1982 e 2011 foram notificados 659 casos de botulismo infantil na Argentina, afetando principalmente crianças com idade inferior a seis meses ${ }^{30}$. A maioria dos casos relatados na literatura ocorreu em pacientes com idade inferior a um ano, atribuindo-se esta característica como o único fator de predisposição reconhecida para o botulismo infantil. Isso porque, a doença parece estar relacionada a uma falha transitória da microbiota intestinal que inibe competitivamente o crescimento dos esporos ${ }^{17}$.
Segundo Fenicia e Anniballi ${ }^{17}$, até o ano de 2009 todos os continentes relataram casos de botulismo infantil, exceto a África. De acordo com os autores, os esporos de Clostridium estão distribuídos no ambiente, comumente presente no solo, produtos agrícolas e poeira doméstica. Dessa forma, bebês expostos repetidamente inalam esses esporos, transportados por partículas microscópicas de poeira.

A incidência e os tipos de toxinas para o botulismo infantil refletem a integridade e a geografia dos esporos no ambiente ${ }^{26}$. As plantas medicinais podem ser contaminadas por esporos botulinicos presentes no ambiente, uma vez que o solo é a principal fonte destes. Nesse sentido, a altura da planta pode ser determinante no processo de contaminação, quando esta cresce próxima do solo. Dessa forma, como medida preventiva à ocorrência de botulismo infantil, deve-se evitar que crianças consumam produtos em que a presença de esporos tenha sido identificada ${ }^{18}$.

Depois dos Estados Unidos, a Agentina apresenta o segundo maior relato de casos de botulismo infantil. Muitos destes foram associados à infusão de ervas, como camomila e hortelã, comumente administrada em lactentes para tratar cólicas intestinais. Essa prática também é comum em outros países da Europa, como Itália e Espanha ${ }^{17}$.

Embora a poeira microscópica do ambiente seja a maior fonte de esporos desse microrganismo, o mel continua sendo o único alimento comprovadamente associado ao botulismo infantil ${ }^{26}$. A padronização dos rótulos das embalagens de méis, advertindo sobre o risco do consumo deste por crianças menores de um ano ajuda a prevenir a doença. Além disso, a conscientização da doença entre os profissionais de saúde deve ser uma prioridade, pois permite um rápido diagnóstico e tratamento adequado, melhorando a notificação e reduzindo possíveis surtos ${ }^{26}$.

\section{CONSIDERAÇÕES FINAIS}

O maior risco de contaminação do botulismo alimentar está nos alimentos preparados artesanalmente, especialmente as conservas caseiras manipuladas de forma inadequada ou que não sofreram tratamento térmico suficiente para destruição dos esporos do Clostridium botulinum. Os casos de botulismo infantil foram notificados em vários países e, embora a principal fonte de risco comprovada seja o consumo de mel por crianças menores de um ano de idade, a maioria dos relatos na literatura identificou a poeira, doméstica ou não, aspirada pelos pacientes como uma provável causa da doença.

Embora o botulismo seja uma doença mundialmente conhecida, as limitações em identifica-la contribuem para o diagnóstico incorreto e a subnotificação da 
doença. Por ser um problema de emergência pública devido à gravidade da doença, faz-se necessário a realização de diagnóstico precoce, a fim de melhorar a notificação e prevenir eventuais surtos. Quanto à precisão dos dados epidemiológicos, infere-se o cuidado no preenchimento de dados relevantes para a notificação de doenças compulsórias pelos profissionais de saúde, bem como salientar a importância da conscientização destes profissionais quanto à interação com a vigilância epidemiológica e sanitária a fim de que o sistema de informação e monitoramento de surtos ocorridos no país seja adequado e eficiente.
Os dados apresentados também demonstram a importância de sensibilizar a população dos principais riscos e medidas de prevenção, já que a maioria dos casos relatados está relacionada a práticas inadequadas de preparo dos alimentos. Ressalta-se também, a importância da realização de investigações na detecção do Clostridium botulinum, tanto em alimentos, quanto na poeira, principalmente no Brasil, devido à abordagem do tema ainda escassa e à dificuldade de encontrar dados na literatura.

\section{Conflitos de Interesse}

Os autores declaram não ter conflitos de interesse.

\section{REFERÊNCIAS}

1. Córdova GL, Escobar RH, Perrel CP, Castillo AM, Carrasco JAO, Rodríguez JI. Botulismo infantil: comunicación de un caso y revisión del tema. Rev Chil Pediatr. 2008;79(4):409-14. http://dx.doi.org/10.4067/S037041062008000400010

2. Silva BRTC, Pessoa NO. Botulismo por Clostridium botulinum na intoxicação alimentar animal e humana. Uma Revisão. Rev Bras Hig Sanid Anim. 2015;9(4):733-47.

3. Mangilli LD, Andrade CRF. Botulismo e disfagia. Pró-Fono $R$ Atual Cient. 2007;19(2):215-222. http://dx.doi.org/10.1590/S010456872007000200010 .

4. Cereser ND, Costa FMR, Rossi OD Jr, Silva DAR, Sperotto VR. Botulismo de origem alimentar. Cienc Rural. 2008;38(1):280-7. http://dx.doi.org/10.1590/S010384782008000100049 .

5. Tornese M, Rossi ML, Coca F, Cricelli C, Troncoso A. Epidemiología y factores de riesgo asociados al botulismo de los alimentos y al botulismo infantil: ¿Dónde y cuándo? Rev Chilena Infectol. 2008;25(1):227. http://dx.doi.org/10.4067/ S0716-10182008000100004 PMid:18273519.

6. Figueiredo MAA, Dias J, Lucena R. Considerações acerca de dois casos de botulismo ocorridos no Estado da Bahia. Rev Soc Bras Med Trop. 2006;39(3):28991. http://dx.doi.org/10.1590/ S0037-86822006000300014 PMid:16906257.
7. Parrilli CC. Clostridium botulinum em alimentos [trabalho de conclusão de curso]. São Paulo (SP): Faculdades Metropolitanas Unidas; 2008.

8. Barboza MMO, Santos NF, Souza OV. Surto familiar de botulismo no Estado do Ceará: relato de caso. Rev Soc Bras Med Trop. 2011;44(3):4002. http://dx.doi.org/10.1590/ S0037-86822011000300030. PMid:21779684.

9. Arriagada DS, Wilhelm JB, Donoso AF. Botulismo infantil. Comunicación de un caso clínico y revisión de la literatura. Rev Chilena Infectol. 2009;26(2):1627. http://dx.doi.org/10.4067/ S0716-10182009000200009. PMid:19621149.

10. Juliano JAF, Cardoso AM. Clostridium botulinum e suas toxinas: uma reflexão sobre os aspectos relacionados ao botulismo de origem alimentar. Rev Est. 2014;41(3):65770.

11. Rowlands REG, Ristori CA, Lopes GISL, Paula AMR, Sakuma H, Grigaliunas R, et al. Botulism in Brazil, 2000-2008: epidemiology, clinical findings and laboratorial diagnosis. Rev Inst Med Trop. 2010;52(4):1836. http://dx.doi.org/10.1590/ S0036-46652010000400003. PMid:21748224.

12. Jalda Sánchez D, Junco García A, Álvarez Moreno M, Rodero Garduño I, Carneado Ruiz J. Microepidemia familiar de botulismo alimentario en la Comunidad de Madrid. Rev Neurol. 2016;63(1):28-32. http:// dx.doi.org/10.33588/rn.6301.2016081. PMid:27345277.
13. São Paulo. Secretária de Estado da Saúde. Botulismo. Orientações para pacientes e familiares. Divisão de doenças de transmissão hídrica e alimentar. São Paulo: Secretária de Estado da Saúde; 2002 [citado 2017 fev 19]. 51 p. Disponível em: http:// www.cve.saude.sp.gov

14. Ragazani AVF, Schoken-Iturrino RP, Garcia GR, Delfino TPC, Poiatti ML, Berchielli SP. Esporos de Clostridium botulinum em mel comercializado no Estado de São Paulo e em outros Estados brasileiros. Cienc Rural. 2008;38(2):396-9. http://dx.doi.org/10.1590/S010384782008000200016

15. Sheth AN, Wiersma P, Atrubin D, Dubey V, Zink D, Skinner G, et al. International outbreak of severe botulism with prolonged toxemia caused by commercial carrot juice. Clin Infect Dis. 2008;47(10):1245-51. http://dx.doi.org/10.1086/592574. PMid:18834318.

16. Bilusic M, Pattathil J, Brescia M, McHugh W, Zaboski M, Schanzer B. Recurrent bulbar paralysis caused by botulinum toxin type B. Clin Infect Dis. 2008;46(8):72-4. http://dx.doi. org/10.1086/533470. PMid:18444842.

17. Fenicia L, Anniballi F. Infant botulism. Ann Ist Super Sanita. 2009;45(2):13446. PMid:19636165.

18. Bianco MI, Lúquez $\mathrm{C}$, De Jong LIT, Fernández RA. Linden flower (Tilia spp.) as potential vehicle of Clostridium botulinum spores in the transmission of infant botulism. Rev Argent Microbiol. 2009;41(4):232-6. PMid:20085187. 
19. Sagua MD, Lúquez $C$, Barzola $C P$, Bianco MI, Fernández RA. Phenotypic characterization of Clostridium botulinum strains isolated from infant botulism cases in Argentina. Rev Argent Microbiol. 2009;41(3):141-7. PMid:19831311.

20. Vugia DJ, Mase SR, Cole B, Stiles $\mathrm{J}$, Rosenberg J, Velasquez L, et al. Botulism from Drinking Pruno. Emerg Infect Dis. 2009;15(1):69-71. http:// dx.doi.org/10.3201/eid1501.081024

21. Zhang S, Wang Y, Qiu S, Dong $\mathrm{Y}, \mathrm{Xu} \mathrm{Y}$, Jiang D, et al. Multilocus outbreak of foodborne botulism linked to contaminated sausage in Hebei Province, China. Clin Infect Dis. 2010;51(3):322-5. http://dx.doi. org/10.1086/653945. PMid:20569065.

22. Swaan CM, Van Ouwerkerk IM, Roest HJ. Cluster of botulism among Dutch tourists in Turkey, June 2008. Euro Surveill. 2010;15(14):1-5. PMid:20394717.

23. Browning LM, Prempeh $\mathrm{H}$, Little C, Houston C, Grant K, Cowden JM, et al. An outbreak of foodborne botulism in Scotland, United Kingdom, November 2011. Euro
Surveill. 2011;16(49):1-2. http://dx.doi. org/10.2807/ese.16.49.20036-en. PMid:22172331.

24. Pingeon JM, Vanbockstael C, Popoff MR, King LA, Deschamps B, Pradel $\mathrm{G}$, et al. Two outbreaks of botulism associated with consumption of green olive paste, France, September 2011. Euro Surveill. 2011;16(49):1-3. http:// dx.doi.org/10.2807/ese.16.49.20035en. PMid:22172329.

25. Derman Y, Korkeala H, Salo E, Lönnqvist T, Saxen H, Lindström $M$. Infant botulism with prolonged faecal excretion of botulinum neurotoxin and Clostridium botulinum for 7 months. Epidemiol Infect. 2014;142(2):335-9. http://dx.doi. org/10.1017/S0950268813001258. PMid:23688392.

26. Hernández-de Mezerville M, Rojas-Solano M, Gutierrez-Mata A, Hernández-Con L, Ulloa-Gutierrez R. Infant botulism in Costa Rica: first report from Central America. $J$ Infect Dev Ctries. 2014;8(1):123-5. PMid:24423723.

27. Momose $\mathrm{Y}$, Asakura $\mathrm{H}$, Kitamura M, Okada Y, Ueda Y, Hanabara $Y$, et al. Food-borne botulism in
Japan in March 2012. Int J Infect Dis. 2014;24:20-2. http://dx.doi. org/10.1016/j.ijid.2014.01.014.

28. Viray MA, Wamala J, Fagan $R$, Luquez C, Maslanka S, Downing $R$, et al. Outbreak of type A foodborne botulism in a boarding school Uganda, 2008. Epidemiol Infect. 2014;142(11):2297-301. http://dx.doi. org/10.1017/S0950268814000387. PMid:24576562.

29. Feng L, Chen X, Liu S, Zhou Z, Yang R. Two-family outbreak of botulism associated with the consumption of smoked ribs in Sichuan Province, China. Int J Infect Dis. [online] 2015 [citado 2017 mar 8];30:74-77. Disponível em: http:// creativecommons.org/licenses/by-nc$\mathrm{nd} / 3.0 /$

30. Spinia RG, Ferrarisa V, Glasmana MP, Orofinoa G, Casanovasb A, Debaisic G. Invaginación intestinal en un lactante con diagnóstico de botulismo. Reporte de un caso. Arch Argent Pediatr. 2015;113(5):286-9.

31. Anniballi $F$, Auricchio B, Fiore A, Lonati D, Locatelli CA, Lista F, et al. Botulism in Italy, 1986 to 2015 . Euro Surveill. 2017;22(24):1-8. http:// dx.doi.org/10.2807/1560-7917. ES.2017.22.24.30550.

Recebido: 19 ago, 2018 Aceito: 15 maio, 2019 